\title{
interview
}

\section{Gain-switching success}

\author{
Photonics currently lacks a way of amplifying terahertz pulses in time-domain spectroscopy. \\ Nathan Jukam from the École Nomale Supérieure in France spoke to Nature Photonics about how his group \\ has achieved a semiconductor-based terahertz amplifier that gives a greatly improved amplification factor.
}

\section{Tell us about your work.}

For the past 10-20 years, many studies have used terahertz (THz) time-domain spectroscopy (TDS) to investigate materials and physical phenomena. The technique uses a near-infrared femtosecond laser to both create and detect $\mathrm{THz}$ radiation. It's a very powerful technique because it measures the $\mathrm{THz}$ electric field as a function of time. The major drawback to this approach, however, is that besides the small field amplitude per unit frequency, a larger TDS signal requires a large-scale expensive configuration of femtosecond lasers. Our most recent work reports how a semiconductor quantum cascade laser (QCL) can amplify the $\mathrm{THz}$ pulses of a TDS system using gain switching. This work has the potential to increase the use of smaller and cheaper fibre-based femtosecond lasers for TDS. These lasers currently offer only weak THz signals, but combining them with gain-switched QCLs for amplification could result in greatly improved systems.

\section{What is the limitation of QCLs for THz TDS?}

Many groups have used THz TDS to study gain in QCLs. However, these THz lasers operate in the steady state, where the gain is exactly equal to the waveguide and mirror losses of the laser. We often say that the laser action 'clamps' the gain to the total losses. This limits the amplification of any external radiation that is coupled to the laser, because the full gain of the laser is not exploited. In fact, for steady-state laser action, the amplification actually depends on the reflectivity of the mirrors and not the gain. This may sound counter-intuitive, but keep in mind that a larger gain results in a larger laser field, which is a very good thing if you are trying to make a better laser. A good laser, however, is not necessarily a good amplifier.

\section{What did you do to overcome this limit? \\ Our approach was to 'unclamp' the gain by placing the laser in a non-equilibrium state, which allows us to exploit the entire gain of the laser. To do this, we turned on the gain of the $\mathrm{THz}$ laser for a very short time - much faster than the build-up}

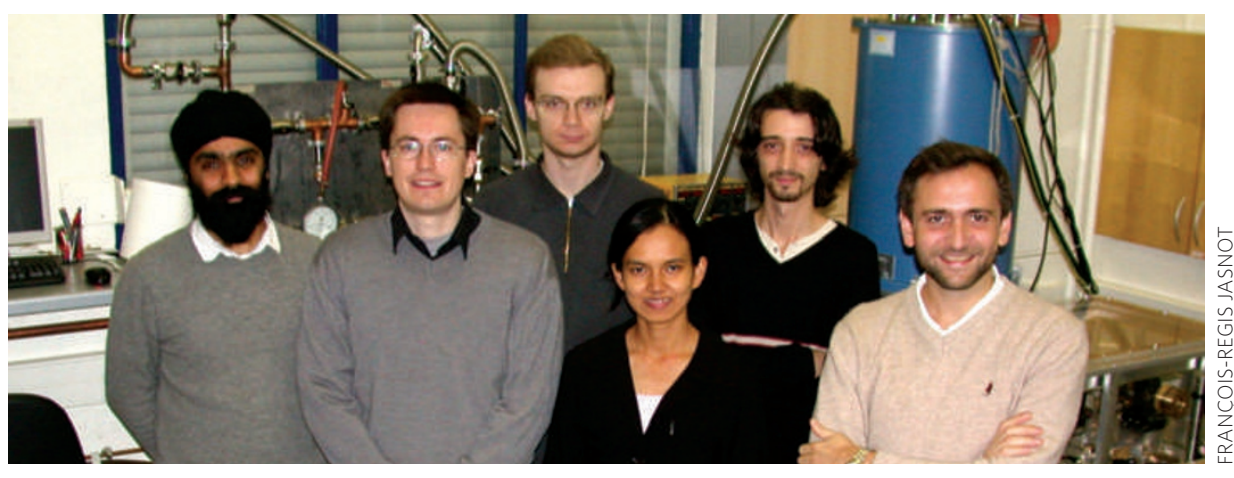

The terahertz spectroscopy group at the École Nomale Supérieure, France. (From left to right: Sukhdeep Dhillon, Nathan Jukam, Dimitri Oustinov, Rakchanok Rungsawang, Julien Madéo and Jérôme Tignon)

time of the laser field - by using an electrical switch operated using an ultrafast femtosecond laser. During the laser buildup time, the gain was greater than the total losses and was therefore unclamped, so the entire gain was used to amplify externally coupled $\mathrm{THz}$ pulses. Turning the gain on quickly to avoid gain clamping is generally referred to as gain switching. It is somewhat analogous to Q-switching, which involves quickly switching the mirror reflectivity (not the gain).

\section{What results have you achieved?}

We obtained large THz power amplification - up to $26 \mathrm{~dB}$, excluding coupling losses - which is well above the limits imposed by the mirror losses and gain clamping. We found that the longer the $\mathrm{THz}$ probe pulses stayed in the laser cavity, and the more round-trips they took inside the laser cavity, the bigger the $\mathrm{THz}$ pulses became. This is the key result of our work, and the signature of unclamped gain. When the gain is greater than both the waveguide and mirror losses, a $\mathrm{THz}$ pulse that makes a round-trip in the cavity will experience a net amplification. Furthermore, our system measures much greater amplifications than previous studies. We show that this makes gain-switched QCLs very good candidates for amplifiers in TDS.

\section{What are the applications?}

Potential and current applications of $\mathrm{THz}$ TDS include, but are not limited to, security and biological imaging, chemical and biological spectroscopy, and the detection of explosives, illicit substances and defects. A practical amplifier could lead to TDS systems that depend little on femtosecond laser performance. If the coupling into the laser can be improved, we should be able to generate a much larger field amplitude per unit frequency, compared with conventional TDS. This could open the door to nonlinear experiments and coherence investigations of narrowband $\mathrm{THz}$ phenomena.

\section{How can the system be improved?}

In some sense, lasers are the ultimate amplifier. When initially turned on they take a spontaneous emission noise signal and amplify it to a very large value. We can imagine replacing the spontaneous emission with a THz pulse from a TDS set-up. In this case, the laser will 'lase' on the $\mathrm{THz}$ pulse. In the present experiments, this did not occur because the electrical pulse was shorter than the laser build-up time. So the next big challenge is to increase the duration and power of the electrical pulse that turns on the QCL laser. Another improvement would be to further reduce the rise time of the electrical pulse, which would allow the gain to be switched on more quickly.

\section{INTERVIEW BY RACHEL WON}

Nathan Jukam and his co-workers have a Letter on semiconductor-based terahertz amplifiers on page 715 of this issue. 